\title{
Spinning continuous carbon nanotube yarns
}

\section{Carbon nanotubes weave their way into a range of imaginative macroscopic applications.}

T he creation of continuous yarns made out of carbon nanotubes would enable macroscopic nanotube devices and structures to be constructed ${ }^{1,2}$. Here we show that carbon nanotubes can be self-assembled into yarns of up to $30 \mathrm{~cm}$ in length simply by being drawn out from superaligned arrays of carbon nanotubes, and that the strength and conductivity of these yarns can be enhanced by heating them at high temperatures. Our findings should help to translate the remarkable mechanical, electrical and thermal properties of carbon nanotubes to a macroscopic scale.

While attempting to pull out a bundle of carbon nanotubes (CNTs) from a CNT array several hundred micrometres high and grown on a silicon substrate, we obtained instead a continuous yarn of pure CNTs (Fig. 1a). This process is very similar to drawing a thread from a silk cocoon, corresponding here to the CNT array. Figure $1 \mathrm{~b}$ shows a $100-\mu \mathrm{m}$-high, freestanding CNT array held by adhesive tape: the indentation at the top of the array marks the region that is being turned into a yarn $30 \mathrm{~cm}$ long and $200 \mu \mathrm{m}$ wide. We estimate that an array area of roughly $1 \mathrm{~cm}^{2}$ can generate about $10 \mathrm{~m}$ of yarn.

Although several methods can be used to prepare CNT arrays on different substrates $^{3-6}$, we find that continuous yarns can only be drawn out from superaligned arrays in which the CNTs are aligned parallel to one another and are held together by van der Waals interactions to form bundles (Fig. 1c). The yarns usually appear as thin ribbons composed of parallel threads that have diameters in the range of several hundreds of nanometres (Fig. 1d), with the width of the yarn roughly depending on the number of threads in the yarn. In principle, the size of the yarn can be controlled by the tip size of the tool that is used to pick up the yarn - the smaller the tip, the thinner the yarn.

To demonstrate the properties of these yarns, we constructed a light-bulb filament by winding a CNT yarn between two metal leads. The filament emits incandescent light when a DC voltage is applied in a vacuum of $5 \times 10^{-3} \mathrm{~Pa}$ (Fig. 1e). After emitting light for $3 \mathrm{~h}$ at $70 \mathrm{~V}$, the conductivity of the filament increases by $13 \%$ and the tensile strength changes from $1 \mathrm{mN}$ to 6.4 $\mathrm{mN}$. These results indicate that some welding effect may be occurring at the weak connection points of the CNTs during light emission, because these

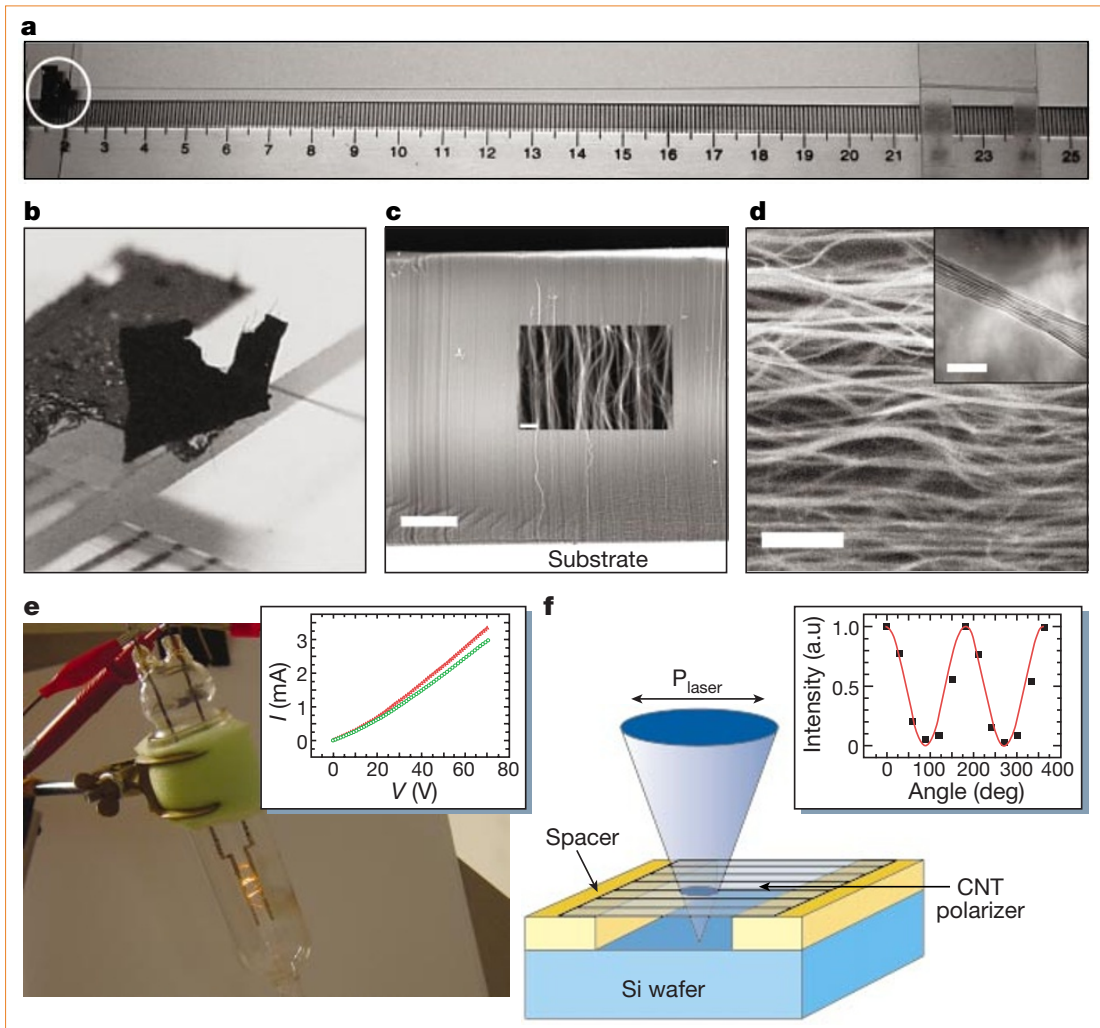

Figure 1 Carbon nanotube yarns. a, b, A carbon nanotube yarn being continuously pulled out from a free-standing carbon nanotube array (a), which is shown enlarged in $\mathbf{b}$ (roughly $\times 8$ magnification). c, Scanning electron microscope (SEM) images of a carbon nanotube array grown on a silicon substrate, showing the superalignment of carbon nanotubes (scale bars: $100 \mu \mathrm{m}$; inset, $200 \mathrm{~nm}$ ). d, SEM image of the yarn in a; inset, transmission electron microscope (TEM) image of a single thread of the yarn (scale bars: 500 $\mathrm{nm}$; inset, $100 \mathrm{~nm}$ ). e, Carbon nanotube filaments emitting incandescent light. Inset, I-V curve measured before (green) and after (red) light emission for $3 \mathrm{~h}$ at $70 \mathrm{~V}$. $\mathbf{f}, \mathrm{A}$ carbon nanotube polarizer. Polarized laser light $\left(\mathrm{P}_{\text {laser }}\right)$ is focused on the surface of a silicon wafer and reflected back, passing the carbon nanotube polarizer twice before being collected by a CCD detector. Inset, normalized intensity (squares, ratio of the current $/$ to $I_{\text {max }}$; a.u., arbitrary units) plotted against the angle between the polarization direction of the laser light and the carbon nanotube polarizer, compared with values calculated from Malus's law $\left(I=I_{0} \cos ^{2} \theta\right.$; red line).

points have a higher resistivity and, as a result, a higher temperature when a current is applied.

We were also able to construct a CNT polarizer by parallel alignment of CNT yarns. When a beam of light passes through the CNT polarizer, photons having a polarization direction parallel to the axis of the CNTs are absorbed, whereas those that are perpendicularly polarized pass through $\mathrm{it}^{7,8}$. Because the CNT diameter is about $10 \mathrm{~nm}$, the polarizer can work in the ultraviolet region, and could even be used for ultraviolet light of wavelengths in the region of tens of nanometres. Figure if shows the polarization ability of the CNT device measured at $325 \mathrm{~nm}$, which is in good agreement with that predicted by Malus's law, $I=I_{0} \cos ^{2} \theta$. The degree of polarization of the CNT polarizer, $P=\left(I_{\max }-I_{\min }\right) /\left(I_{\max }+I_{\min }\right)$, is 0.92 .
We envisage that pure CNT yarns such as these, particularly after appropriate heat treatment, should eventually be able to be woven into a variety of macroscopic objects for different applications, such as bulletproof vests and materials that block electromagnetic waves.

Kaili Jiang, Qunqing Li, Shoushan Fan Department of Physics and Tsinghua-Foxconn Nanotechnology Research Center, Tsinghua University, Beijing 100084, China e-mail: fss-dmp@mail.tsinghua.edu.cn

\footnotetext{
1. Vigolo, B. et al. Science 290, 1331-1334 (2000). 2. Ball, P. Nature 414, 142-144 (2001).

3. de Heer, W. A. et al. Science 268, 845-847 (1995)

4. Li, W. Z. et al. Science 274, 1701-1703 (1996).

5. Ren, Z. F. et al. Science 282, 1105-1107 (1998)

6. Fan, S. S. et al. Science 283, 512-514 (1999).

7. Wang, J. F., Gudiksen, M. S., Duan, X. F., Cui, Y. \& Lieber, C. M Science 293, 1455-1457 (2001).

8. Li, Z. M. et al. Phys. Rev. Lett. 87, 1274011-1274014 (2001).

Competing financial interests: declared none
} 